\title{
Influence of sex on aquaporin 1-4 and vasopressin V2 receptor expression in the pig kidney during development
}

\author{
Lu Xing' and Rikke Nørregaard'
}

BACKGROUND: The ability of the immature kidney to concentrate urine is lower than in adults. The aquaporin (AQP) family and the vasopressin $\mathrm{V} 2$ receptor $(\mathrm{V} 2 \mathrm{R})$ play a critical role in the urinary concentrating capacity. Here we investigated a possible sex difference in AQP1, AQP2, AQP3, and AQP4 as well as $V 2 R$ expression in the fetal pig kidney at different gestation stages.

METHODS: Pig fetuses were divided into three groups according to gestation age of 60, 80, and $100 \mathrm{~d}$. Quantitative PCR and immunohistochemistry were used to determine the regulation of AQP1, AQP2, AQP3, and AQP4 as well as V2R in the fetal pig kidneys.

RESULTS: Renal AQP1, AQP2 and AQP3, and V2R expression was increased with gestation age in both sexes, whereas AQP4 expression was unchanged over time. We observed neither sex differences in the AQPs nor V2R expression in the fetal pig kidneys.

CONCLUSION: AQP1, AQP2, and AQP3, and V2R expression increased with gestation age in the fetal kidney, suggesting that this induction might contribute to the maturation of urinary concentrating capacity. However, no sex differences were observed indicating that sex might not play a role for the maturation of the urinary concentrating activity during kidney development in fetal pig.

A quaporins (AQPs) are a family of water channels that facilitate water transport across the plasma membranes. In the kidney, AQP1 plays a role in constitutive water reabsorption in proximal tubules and AQP2, AQP3, and AQP4 contribute to urinary concentrating capacity in the collecting ducts (1). The major factor of water homeostasis in animals and humans is the regulation of water excretion by circulating plasma levels of the arginine vasopressin (AVP) (2).

It is known that during the development of mammalian, the fetal kidney produces a relatively large volume of hypotonic urine, which is of vital importance for the development of the fetus. It has been suggested that the relative large urine flow $(0.3 \mathrm{l} / \mathrm{kg} / \mathrm{d}$ in fetal sheep vs. $0.02 \mathrm{l} / \mathrm{kg} / \mathrm{d}$ in the adult) might be because of the reduced sensitivity of the fetal kidney to AVP and the relative insensitivity of AVP release to osmotic stimulation (3). In addition, studies have demonstrated that prolonged AVP infusion in fetal lambs can increase amniotic fluid osmolality (4), indicating that circulating AVP modulates fetal renal function and amniotic fluid volume. AVP binds to the vasopressin V2 receptor (V2R) in the kidney which results in increased water permeability of the collecting duct through the insertion of AQP2 into the apical membranes of collecting duct principal cells (1). V2R transcript has been found at $16 \mathrm{~d}$ gestational age in the rat kidney and V2R mRNA is mainly detected in cells of developing medullary and cortical collecting ducts (5). In addition, low levels of AQP2 have been detected in the fetal kidney which appears to be the key factor in allowing the production of large volume of hypertonic urine to be formed and this is crucial for the maintenance of sufficient volumes of amniotic fluid (6).

Previous studies have demonstrated that in human and sheep fetus, renal AQP1 and AQP2 were expressed at wk 12 (gestation period $40 \mathrm{wk}$ ) and at day 40 (gestation period 145$150 \mathrm{~d}$ ), respectively. In the rats, AQP1 and AQP2 mRNA and protein as well as AQP3 mRNA are expressed relatively later at d 18 (gestation period $21 \mathrm{~d}$ ), and the AQP4 mRNA is almost undetectable in the fetal rat kidney $(1,7,8)$. This indicates that the renal AQPs developed earlier in human and sheep than in rats and mice.

To our knowledge, there is no data on sex differences in renal AQP1, AQP2, AQP3, and AQP4 as well as V2R expression in the developing kidney. The upper urinary tract as well as kidney architecture and function in the pig are similar to those in humans (9). Consequently, the pigs are considered to be a suitable animal model for studying the underlying physiological mechanism of AQPs expression in the human fetal kidney. Thus, the aim of this study was to investigate a possible sex difference in AQP1, AQP2, AQP3, and AQP4, as well as $\mathrm{V} 2 \mathrm{R}$ expression in the pig fetal kidney in different periods of gestation.

\section{RESULTS}

\section{Sex Determination of Pig Fetuses}

PCR analysis of the Y-chromosome-specific gene (Sry) sequence generated a 131 base pair (bp) product in the male pig kidney but not in the female one (Figure 1a), and X-chromosome-specific 
gene (Daxx) sequence generated a $179 \mathrm{bp}$ in both male and female pig kidneys (Figure 1b). Twenty nine fetal pigs were determined as males; seven in G60, eight in G80, and 14 in G100; and 17 fetal pigs were determined as females, six in G60, seven

a

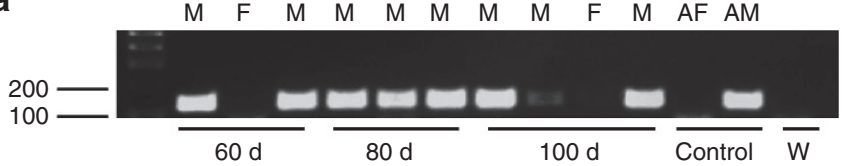

b

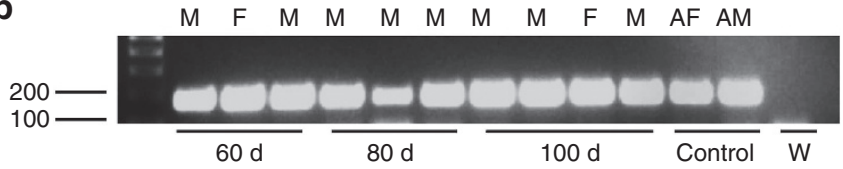

Figure 1. Representative figure showed polymerase chain reaction (PCR) sex typing of fetal pigs. (a) Sry (Y-chromosome-specific gene) detecting a 131-base pair PCR product indicating the male (M) sex. The absence of the Sry product indicates female (F) sex. An adult male (AM) and an adult female (AF) were used as positive controls and water (W) was used as a negative contamination control. (b) Daxx (X-chromosome-specific gene) detecting a 179-base pair PCR product showing bands in all samples except the water control.

a

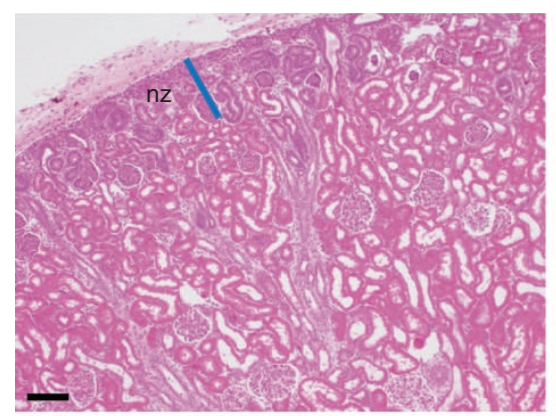

C

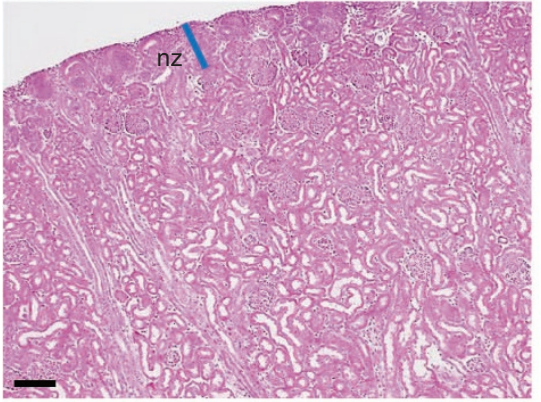

e

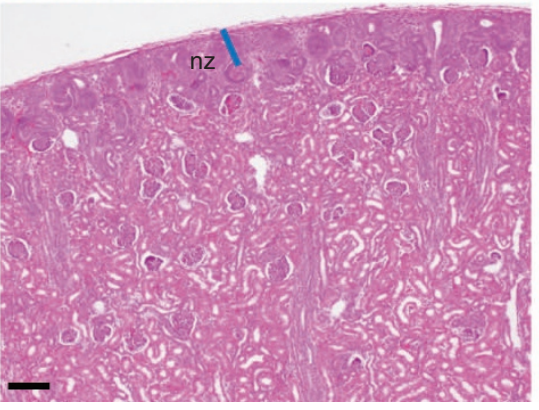

in G80, and four in G100. Furthermore, the sex ratio among the 46 fetuses corresponded to the morphological examination which was performed when the fetuses were dissected.

\section{Morphology of Fetal Pig Kidney in Development}

As shown in Figure 2, the nephrogenic zone appeared smaller in renal cortex from 60 to $100 \mathrm{~d}$ of gestation. In the kidney of $100 \mathrm{~d}$ of gestation, the cortex contained less interstitial tissue due to the proliferation of glomeruli and tubules during the development. The glomeruli at $60 \mathrm{~d}$ of gestation were larger than that at 80 or $100 \mathrm{~d}$ (Figure 2). At $60 \mathrm{~d}$ of gestation, the medulla was rich in interstitial tissue (Figure 2a,b). From 60 to $100 \mathrm{~d}$ of gestation, the medulla got more renal tubules and capillary networks as nephrons developed (Figure 2). There seemed to be no difference in renal morphology and histology between sexes in each group.

\section{Renal mRNA Expression of AQP1, AQP2, AQP3, and AQP4 as well} as V2R During Kidney Development in Fetal Pigs

Quantitative PCR (QPCR) revealed that renal AQP1, AQP2, AQP3, and AQP4 mRNA transcripts were detectable in $60 \mathrm{~d}$ gestation fetuses and the expression increased significantly

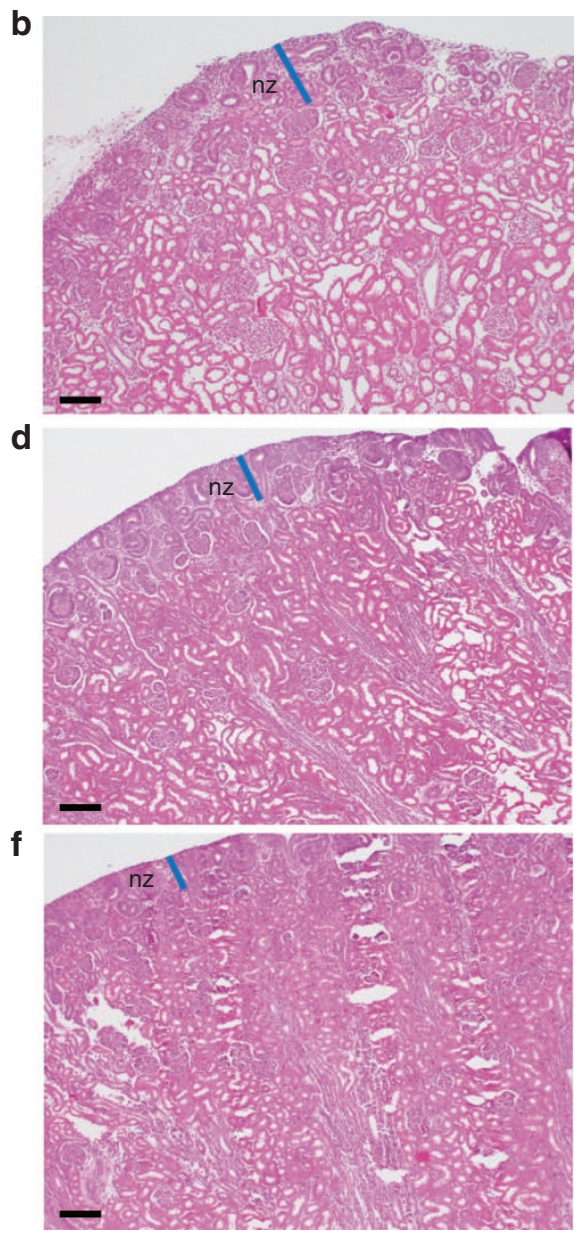

Figure 2. Hematoxylin and Eosin (HE) staining of renal cortex from male and female fetal pigs. Representative images for HE staining in the fetal pig kidney of (a) male at $60 \mathrm{~d}$ of gestation, (b) female at $60 \mathrm{~d}$ of gestation, (c) male at $80 \mathrm{~d}$ of gestation, (d) female at $80 \mathrm{~d}$ of gestation, (e) male at $100 \mathrm{~d}$ of gestation, and (f) female at $100 \mathrm{~d}$ of gestation. The maturation process was shown by the decrease in the nephrogenic zone ( $\mathrm{nz}$ ) (blue line) with gestation stage. Original magnification: 10×. Scale bar: $100 \mu \mathrm{m}$. 
a

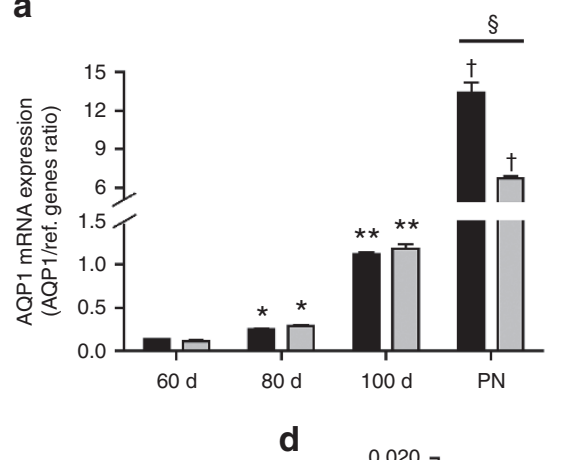

b

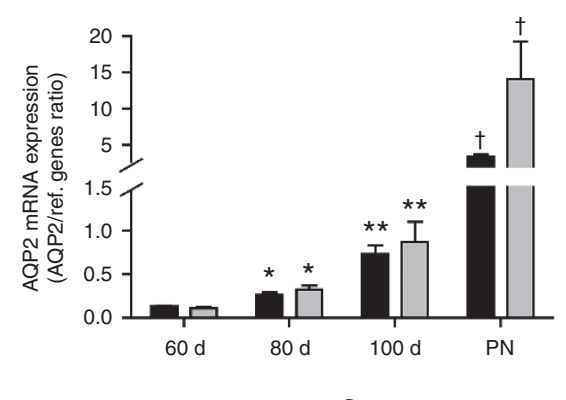

C

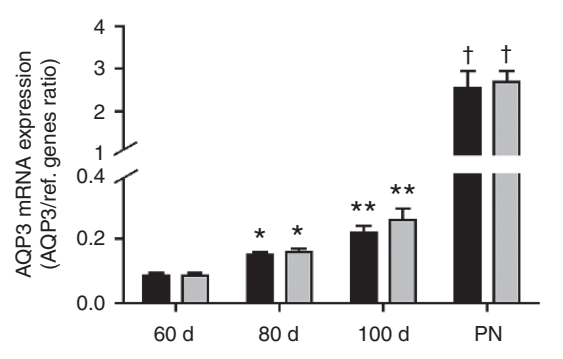

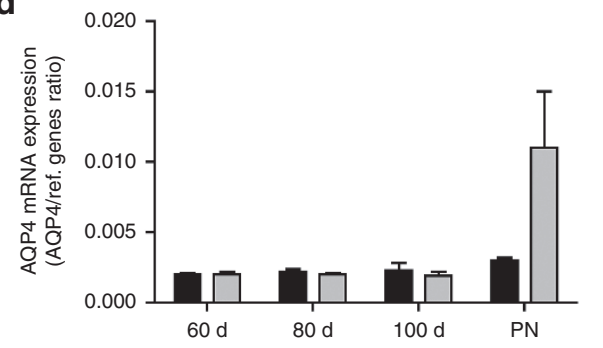

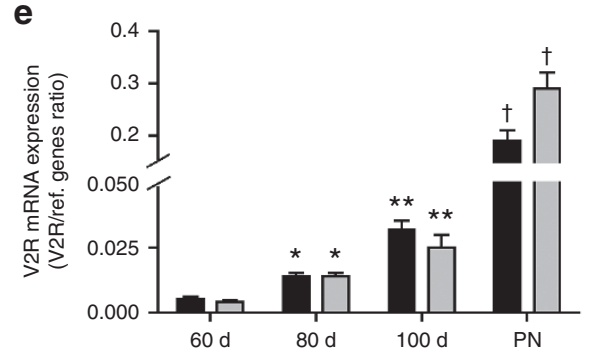

Figure 3. The expression of aquaporin (AQP) 1, AQP2, AQP3, and AQP4 as well as Vasopressin V2 receptor (V2R) in fetal and postnephrogenesis pig kidneys. Bar graphs showing developmental expression of renal (a) AQP1, (b) AQP2, (c) AQP3, (d) AQP4, and (e) V2R in male and female pigs from 60, 80, and $100 \mathrm{~d}$ of gestation. The mRNA expression for all measured genes was normalized by the geometrical mean of four reference genes, including $\beta$-actin, glyceraldehyde-3-phophate dehydrogenase (GAPDH), hypoxanthine phosphoribosyltransferase 1 (HPRT1), and TATA box binding protein (TBP). Black bars indicate male and gray bars indicate female. ${ }^{*} P<0.05$ for Day 60 vs. Day80; ${ }^{* *} P<0.05$ for Day 80 vs. Day $100 ;{ }^{\dagger} P<0.05$ for Day 60 , Day 80 , and Day 100 compared with postnephrogenesis; ${ }^{\varsigma} P<0.05$ for male compared with female. PN, postnephrogenesis; Ref. genes, Reference genes.

Table 1. RNA transcription levels (corrected $C_{t}$ values) of the candidate reference genes

\begin{tabular}{|c|c|c|c|c|c|c|c|c|}
\hline \multirow[b]{2}{*}{ Gestation stage } & \multicolumn{2}{|c|}{$\beta$-actin } & \multicolumn{2}{|c|}{ GAPDH } & \multicolumn{2}{|c|}{ HPRT1 } & \multicolumn{2}{|c|}{$T B P$} \\
\hline & Male & Female & Male & Female & Male & Female & Male & Female \\
\hline $60 d$ & $13.2 \pm 0.05$ & $13.2 \pm 0.09$ & $17.2 \pm 0.03$ & $17.2 \pm 0.05$ & $21.1 \pm 0.06$ & $21.0 \pm 0.06$ & $21.8 \pm 0.05$ & $21.8 \pm 0.05$ \\
\hline $100 d$ & $13.5 \pm 0.08$ & $13.3 \pm 0.10$ & $17.6 \pm 0.05$ & $17.7 \pm 0.11$ & $21.4 \pm 0.05$ & $21.4 \pm 0.11$ & $21.9 \pm 0.07$ & $21.9 \pm 0.07$ \\
\hline Adult & $15.5 \pm 0.05$ & $14.4 \pm 0.37$ & $18.3 \pm 0.16$ & $18.4 \pm 0.40$ & $22.6 \pm 0.36$ & $22.5 \pm 0.32$ & $23.2 \pm 0.16$ & $23.2 \pm 0.35$ \\
\hline
\end{tabular}

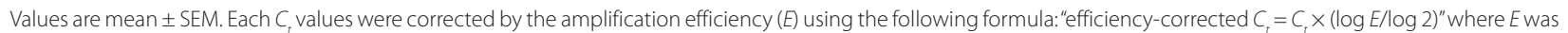
determined using the standard curve for each reference gene.

GAPDH, glyceraldehyde-3-phophate dehydrogenase; HPRT1, hypoxanthine phosphoribosyl-transferase 1; TBP, TATA box binding protein.

from Day 60 to Day 100 of gestation (Figure 3a-c). However, AQP4 mRNA was expressed at very low levels and did not change within the kidney development (Figure 3d). The mRNA expression of the V2R was also very low at Day 60 of gestation but gradually increased over time (Figure 3e). The mRNA expression of AQP1, AQP2, AQP3, and V2R increased significantly in the postnephrogenesis pig kidneys compared with that observed in the fetal pig kidneys (Figure 3a-e). We observed no sex differences in AQP1, AQP2, AQP3, and AQP4 as well as V2R expression in the fetal pig kidneys (Figure 3a-e).

In order to have reliable reference genes for normalization of the genes of interest in the fetal pig kidneys, we selected four genes, including $\beta$-actin, glyceraldehyde-3-phophate dehydrogenase, hypoxanthine phosphoribosyltransferase 1 , and TATA box binding protein which has previously been described as good reference genes for pig kidney tissue (10). There was no significant regulation of the reference genes among the different gestation ages or sex (Table 1). The mRNA expression for all measured genes was normalized by the geometrical mean of all four reference genes.

\section{Localization of AQP1 and AQP2 in Kidneys During Development in Fetal Pigs}

Immunohistochemical staining showed weak labeling of AQP1 in a few proximal tubules at Day 60 in both male and female animals (Figure 4a,b). At day 80 and 100, the staining of AQP1 appeared to increase in intensity gradually over time (Figure $4 \mathrm{c}-\mathrm{f}$ ). The amount of labeling tubules and labeling intensity seemed not to have changed between sexes at any of the time point of the gestation (Figure $4 a-f$ ). In the control postnephrogenesis kidney, stronger labeling of AQP1 protein in the proximal tubules was observed (Figure 4g).

For AQP2, the immunohistochemical staining was very weak and only detectable in a few cells in the collecting ducts at Day 60 (Figure 5a,b). At Day 80 (Figure 5b-d), the AQP2 labeling appeared in more collecting ducts and the staining 

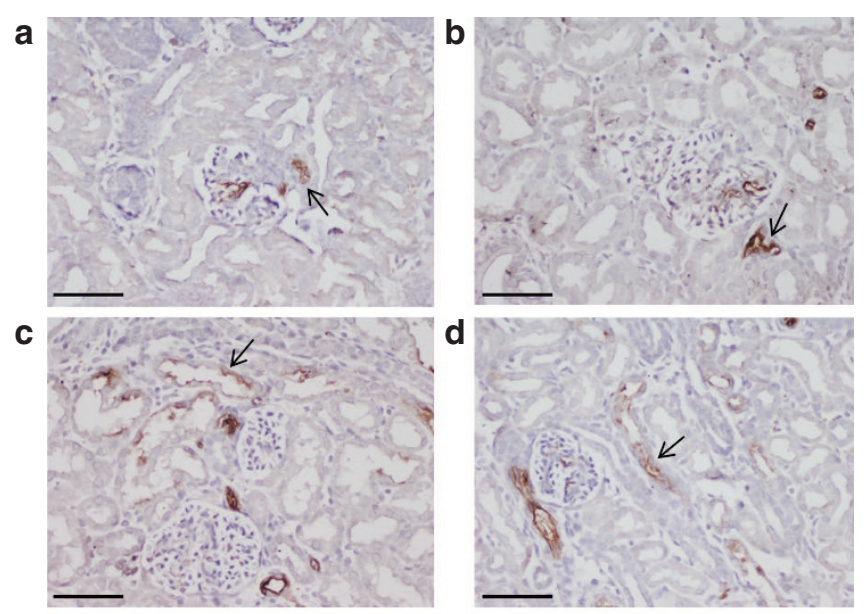

e
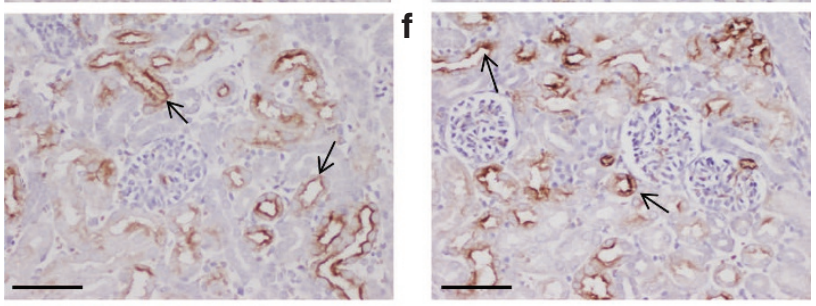

g

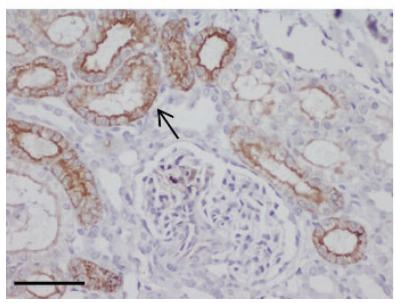

Figure 4. Immunohistochemical staining of aquaporin (AQP)1 in kidneys from fetal and postnephrogenesis pigs. Representative images for AQP1 staining in the fetal pig kidney of (a) male at $60 \mathrm{~d}$ of gestation, (b) female at $60 \mathrm{~d}$ of gestation, (c) male at $80 \mathrm{~d}$ of gestation, (d) female at $80 \mathrm{~d}$ of gestation, (e) male at $100 \mathrm{~d}$ of gestation, (f) female at $100 \mathrm{~d}$ of gestation, and (g) postnephrogenesis pig. Immunohistochemical staining showed localization of AQP1 in the proximal tubules as indicated by arrows and the labeling intensity seemed stronger in Day 100 pigs compared with Day 80, and Day 60 pigs. Original magnification: $40 \times$. Scale bar: $50 \mu \mathrm{m}$.

intensity was stronger than that at Day 60 and even stronger staining was observed at $100 \mathrm{~d}$ of gestation (Figure $5 \mathrm{e}, \mathrm{f}$ ). AQP2 staining intensity seemed not to have changed between sexes at any of the gestation ages investigated (Figure 5a-f). The labeling of AQP2 in the control postnephrogenesis kidney appeared much stronger compared to fetal kidneys (Figure 5g).

\section{Plasma Osmolality in Fetal Pigs}

Because of hemolysis of the plasma samples from the pigs of $60 \mathrm{~d}$ of gestation, we were only capable of analyzing the plasma osmolality from the Day 80 and Day 100 animals (Table 1). The plasma osmolality was significantly increased at Day 100 compared with Day 80 in both sexes, without significant difference between sexes in each group (Table 2).

\section{DISCUSSION}

This study demonstrated that renal AQP1, AQP2, AQP3, and $\mathrm{AQP} 4$ transcripts were detectable in fetal pigs. AQP1, AQP2,
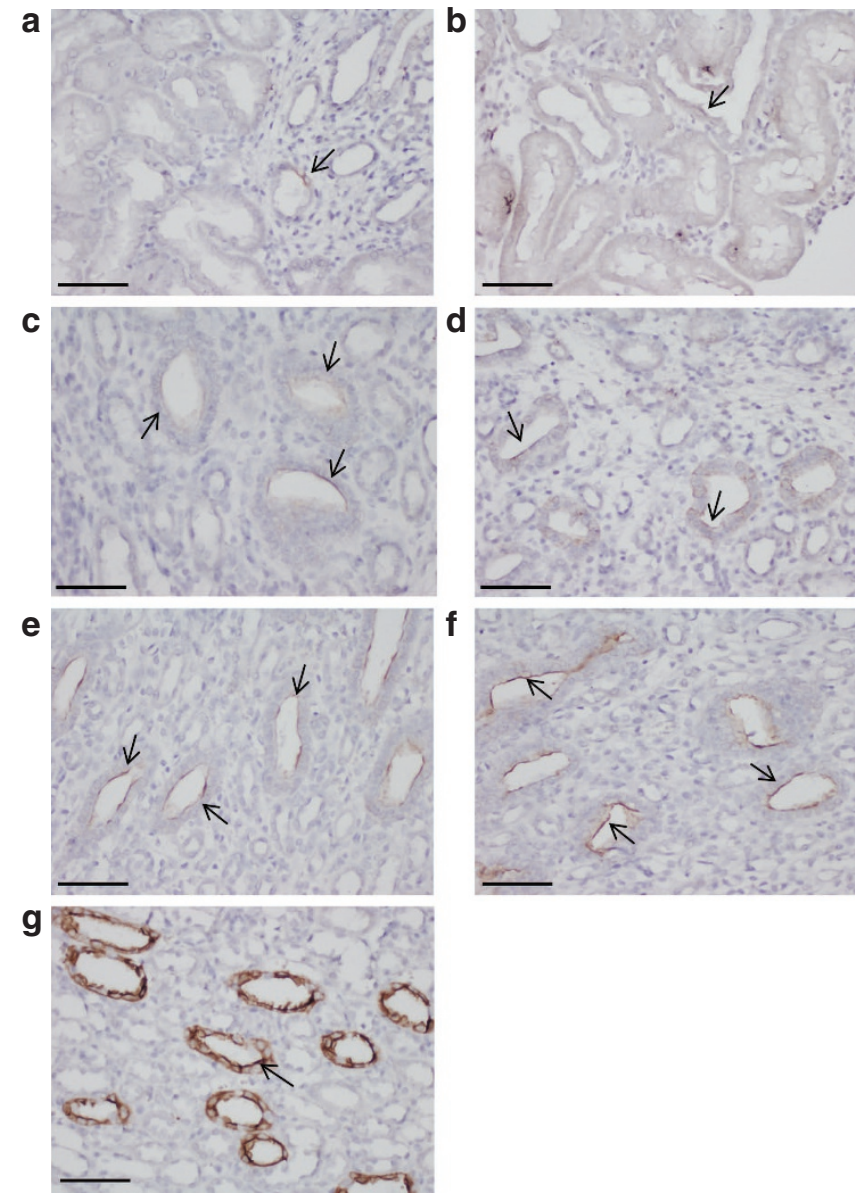

Figure 5. Immunohistochemical staining of aquaporin (AQP) 2 in the kidneys from fetal and postnephrogenesis pigs. Representative images for AQP2 staining in the fetal pig kidney of (a) male at $60 \mathrm{~d}$ of gestation, (b) female at $60 \mathrm{~d}$ of gestation, (c) male at $80 \mathrm{~d}$ of gestation, (d) female at $80 \mathrm{~d}$ of gestation, (e) male at $100 \mathrm{~d}$ of gestation, (f) female at $100 \mathrm{~d}$ of gestation, and (g) postnephrogenesis pig. Immunohistochemical staining showed localization of AQP2 in the collecting ducts as indicated by arrows and the labeling intensity seemed stronger in Day 100 pigs compared to Day 80 and Day 60 pigs. Original magnification: $40 \times$. Scale bar: $50 \mu \mathrm{m}$.

and AQP3 expression increased gradually with gestation age whereas the expression of AQP4 stayed low and was not changed over time. In addition, V2R mRNA expression was very low at Day 60 of gestation but gradually increased over time. We observed no significant sex differences at the investigated stages of gestation.

It has previously been demonstrated in rats that $\mathrm{AQP} 1$ and AQP2 are detectable at embryonic day 16 and AQP3 has been detectable at embryonic day 18 , whereas AQP4 is nearly undetectable in the fetal rat kidney $(1,8,11)$. In fetal kidneys from human and sheep, AQP1 and AQP2 have been detected before midgestation (12/40 wk, human; $41 / 150 \mathrm{~d}$, sheep) (12-14). Our study showed in pig kidneys, that AQP1, AQP2, AQP3, and AQP4 were all detectable in midgestation, earlier than that in the rodent kidneys $(1,8,11)$. In addition, we showed expression of V2R in midgestation in the fetal kidney which increased over time. Earlier data in fetal rat kidneys has demonstrated that V2R mRNA is present as early as embryonic day 16 in the 


\section{Articles | Xing and Norregaard et al.}

Table 2. Plasma osmolality of fetal pigs

\begin{tabular}{lcc}
\hline \multirow{2}{*}{ Gestation stage } & \multicolumn{2}{c}{ Plasma osmolality } \\
\cline { 2 - 3 } $80 \mathrm{~d}$ & $293 \pm 1.9^{*}$ & Female \\
$100 \mathrm{~d}$ & $305.5 \pm 2.0$ & $296.6 \pm 0.8^{*}$ \\
\hline
\end{tabular}

Values are mean \pm SEM. $* P<0.05$ for $80 \mathrm{~d}$ of gestation compared with $100 \mathrm{~d}$ of gestation

collecting ducts with increasing expression of V2R mRNA in the first postnatal weeks (5). Metanephric kidney development varies in different species, first appearing earlier in human $(12 \%$ of gestation), sheep ( $18 \%$ of gestation), and pigs ( $19 \%$ of gestation), later in mice (55\% of gestation) and rats ( $57 \%$ of gestation) (15). Taken together, this might explain the difference in the detection time of AQP1, AQP2, AQP3, and AQP4 as well as $\mathrm{V} 2 \mathrm{R}$ among species in the developing kidney.

The implications of sex difference on the expression of renal AQP1 and AQP2 has only been reported in adult and prepuberty, in rats (16) and humans (17), respectively. To our knowledge, there are no studies about the sex differences in the expression of $\mathrm{AQP} 1, \mathrm{AQP} 2, \mathrm{AQP} 3$, and AQP4 in fetal pig kidneys. Herak-Kramberger et al. (16) reported that in adult rats, the renal AQP1 expression is higher in male compared with female rats but there is no significant difference between sexes in newborn rats, indicating that the male-dominant AQP1 expression may not be developed before puberty. This is consistent with our findings in this study showing increased AQP1 expression in the postnephrogenesis male pig compared with females and no sex difference in AQP1 expression was observed in the fetal pig kidneys from midgestation. In addition, in adult gonadectomized rats, renal AQP1 expression was upregulated strongly by testosterone but moderately by progesterone treatment, indicating that $\mathrm{AQP} 1$ expression might be affected more by androgens then by progesterone (16). Furthermore, Mahler et al. (17) demonstrated that plasma testosterone levels are not changed between sexes in prepuberty children suggesting that sex hormones might not influence AQP1 expression at the prepuberty stage which might also be the case during fetal development.

Circulating AVP can modulate ovine fetal renal function and amniotic fluid volume (4), previous studies have demonstrated that infusion of a selective V2R agonist (desamino, D-Arg8)-AVP changes urine flow to the same level as AVP alone (18), suggesting that the V2R plays an important role during fetal development. AVP binding to the V2R increases water permeability by modulating cell surface expression of AQP2 (19-21). Our data showed no sex-dependent difference in V2R mRNA expression at the investigated days of gestation, but our postnephrogenic data demonstrated a tendency to increased V2R expression in the female pigs. Liu et al. (22) showed in adult rats that females express more V2R mRNA in kidneys than males and suggested that this results physiologically in a greater sensitivity to endogenously secreted AVP or V2R agonist administration in females. However, Wang et al. demonstrated that levels of V2R were higher in male rats (23).
In contrast, another study using spiny mice, which is the only known rodent species that can complete nephrogenesis before birth, found that the expression of V2R did not differ between sexes (24). Taken together, studies report conflicting results using different animal species, so indeed further work to examine the role of V2R between sexes is warranted.

AQP2 expression was not significantly different between male and female fetal pigs over time. This is consistent with a previous study, where they also found no sex-dependent difference in AQP2 expression in adult rats (22). It has been demonstrated that estradiol plays a role for the regulation of AQP2 in female rats subjected to ovariectomy (25). In addition, it has been shown that in human fetuses, the estradiol levels are higher in females than males (26) and moreover data has demonstrated that estradiol can lower plasma AVP levels suggesting that females might have lower AVP levels $(27,28)$. However, men have reduced renal sensitivity to AVP compared with women (29). Taken together, this suggests that the sex-independent AQP2 expression in fetal pig kidneys could be a result of increased estradiol and different renal sensitivity to AVP between sexes. This might also explain the sex-independent regulation of AQP3 and AQP4 mRNA expression in fetal kidneys, as AQP3 and AQP4 expression is regulated by AVP as well (30). Our findings suggest that sex had no effect on AQP3 expression in fetal pig kidneys, is in accordance with previous findings showing no significant difference of AQP3 expression between male and female adult spiny mice (24).

Previous study demonstrated that plasma osmolality is mainly dependent on the homeostatic control of water (31), which is followed by fine-tuning of AQP2 trafficking and expression (32). This study showed that plasma osmolality and AQP2 had the similar expression patterns between sexes and with gestation stage, indicating that AQP2 expression in fetal pig kidneys might play a role in the sex-independent plasma osmolality in fetal development.

In clinical practice, women generally have a lower risk for developing chronic kidney disease than men, in addition, they progress slower to end stage renal disease after renal injury (33). However, the mechanism of sex difference in kidney disease is still unclear. Renal AQPs play an important role in urine concentrating capacity and as result a number of chronic kidney disease produce changes in the abundance of AQPs, such as a reduction in AQP2 expression in various forms of chronic kidney disease (34). We have previously demonstrated, that sex difference effects renal AQP2 expression in response to partial unilateral ureteral obstruction in new born rats showing that AQP2 was downregulated in males, compared with females (35). However, the data on the sex difference of AQPs expression in the developing kidney are very limited.

In conclusion, AQP1, AQP2, and AQP3 as well as V2R expression increased with gestation age in the fetal pig kidney suggesting that this induction might contribute to the maturation of urinary concentrating capacity. However, no sex differences were observed indicating that sex might not play a role for this maturation of the urinary concentrating activity during kidney development in the fetal pig. 
Table 3. Primer sequences

\begin{tabular}{|c|c|c|}
\hline Gene & Sense & Anti-sense \\
\hline$A Q P-1$ & TTG GGC TGA GCA TTG CCA CGC & CAG CGA GTT CAG GCC AAG GGA GTT \\
\hline$A Q P-2$ & CTG TGG AGCTTTTCCTGA CC & TAG TGG ATC CCG AGA AGG TG \\
\hline$A Q P-4$ & GCTTTCTGG AAG GCA GTC AC & CAA TGC TGA GTC CAA AGC AA \\
\hline$V 2 R$ & СTT CTT CCT CGT GCA GCT CT & CGT AGA TCC AGG GGTTGGTA \\
\hline GAPDH & GGG CAT GAA CCA TGA GAA GT & TGT GGT CAT GAG TCC TTC CA \\
\hline HPRT1 & AAG CTT GCT GGT GAA AAG GA & GTC AAG GGC ATA GCC TAC CA \\
\hline$T B P$ & GCC AGA GTT GTTTCC TGG TT & TCG TCTTCCTGA AAC CCTTT \\
\hline Sry & GGA TCG TGT CAA GCG ACC CAT GAA C & GCA TTTTCC ACTTGC ATC CCA GCC \\
\hline Daxx & ACA TCC AGG GAC TTC AGT GGG GAAC & CAT GCT GAC AGT GCC AAT GAT GGG \\
\hline
\end{tabular}

\section{METHODS}

\section{Animals}

All procedures conformed with the Danish National Guidelines for care and handling of animals and to the published guidelines from the National Institutes of Health. The experimental animal protocols were according to the licenses for use of experimental animals issued by The Ministry of Food, Agriculture, and Fishes; The Animal Experiments Inspectorate (Approval no. 2013/15-2934-00779).

A total of 46 pig fetuses from three time-dated pregnant Danish Landrace sows (Research Centre Foulum, Aarhus University, Denmark) were used in the study. The pregnant sows were anesthetized with intraperitoneal injection of pentobarbital. The abdomen was opened, the uterus was removed, and individual gestational sacs were dissected. Both kidneys of the fetus were harvested.

\section{Experiment Protocol}

The fetuses were divided into three groups according to gestation age: G60 ( $n=13$ and $60 \mathrm{~d}$ of gestation), G80 ( $n=15,80 \mathrm{~d}$ of gestation), and G100 ( $n=18100 \mathrm{~d}$ of gestation). The left kidneys from the three groups were used for QPCR, immunohistochemistry and haematoxylin and eosin staining. The right kidneys were used for sex determination. Blood samples from the fetal pigs were used for biochemical analysis. As references, kidneys from five female and three male postnephrogenesis pigs were performed with the same procedure as fetuses.

\section{Extraction of Genomic DNA}

Genomic DNA was extracted from right kidney tissues using lysis buffer with $200 \mathrm{mmol} / \mathrm{l} \mathrm{NaCl}, 100 \mathrm{mmol} / \mathrm{l}$ Tris-Cl, $5 \mathrm{mmol} / \mathrm{l}$ EDTA, $0.2 \%$ sodium dodecyl sulfate, and $10 \mathrm{mg} / \mathrm{ml}$ proteinase $\mathrm{K}$ overnight at $55^{\circ} \mathrm{C}$. DNA was precipitated by isopropanol, washed by $70 \%$ ethanol and then resuspended in $100 \mu \mathrm{l}$ Tris-EDTA buffer.

\section{PCR Analysis of Extracted DNA}

Due to the inherent difficulties in visual sex determination of fetal pigs, a PCR-based assay for unequivocal sex determination was performed. A Sry sequence (male-specific gene on the Y chromosome) and a Daxx sequence (X-chromosome-specific) were amplified by PCR respectively using 10 pmol Sry primers or Daxx primers (Table 3). The following reaction procedure: $3 \mathrm{~min}$ at $94{ }^{\circ} \mathrm{C}$; followed by 34 cycles of $30 \mathrm{~s}$ at $94^{\circ} \mathrm{C}, 30 \mathrm{~s}$ at $60^{\circ} \mathrm{C}$, and $30 \mathrm{~s}$ at $72^{\circ} \mathrm{C}$. Expected band sizes were $131 \mathrm{bp}$ (Sry) and $179 \mathrm{bp}$ (Daxx). Adult male and female pigs were included as positive controls, as well as distilled water were considered as a negative control. PCR products were analyzed by electrophoresis in a $2 \%$ agarose gel at $100 \mathrm{~V}$ for $30 \mathrm{~min}$.

\section{Quantitative PCR}

The left kidney from G60, G80, and G100 fetal pigs were collected. Total RNA was isolated with a NucleoSpin RNA II mini kit following the manufacturer's instructions (Macherey-Nagel, Düren, Germany). RNA was quantitated by spectrophotometry and stored at $-80^{\circ} \mathrm{C}$. cDNA was synthesized from $0.5 \mu \mathrm{g}$ RNA with the AffinityScript QPCR cDNA synthesis kit (Life Technologies, Thermo Fisher Scientific, Cambridge, MA). For quantitative PCR, $100 \mathrm{ng}$ cDNA served as a template for PCR amplification using Brilliant SYBR Green QPCR Master Mix according to the manufacturer's instruction (Life Technologies). Samples were amplified in duplicate in 96-well plates, and PCR was performed for 40 cycles consisting of denaturation for $30 \mathrm{~s}$ at $95{ }^{\circ} \mathrm{C}$ followed by annealing and polymerization at $60{ }^{\circ} \mathrm{C}$ for $1 \mathrm{~min}$. Emitted fluorescence was detected during the annealing/extension step in each cycle. The primer sequences were used according to Table 3. A melting curve analysis was performed, which for all primer sets resulted in single product-specific melting curves with no primer-dimers. A standard curve was constructed by plotting threshold cycle ( $C_{t}$ values) against serial dilutions of cDNA to calculate the individual real-time PCR amplification efficiency $\left(E=10^{(-1 / \text { slope })}\right.$. The relative expression ratio of a target gene was based on its amplification efficiency $(E)$ and the crossing point difference $\left(\Delta C_{\mathrm{t}}\right)$ for an unknown sample vs. a control. The geometric mean of the reference genes was used to correct the raw value of the genes of interest (36).

\section{Immunohistochemistry}

Kidneys from G60, G80, and G100 fetal pigs were immersion fixed for $4 \mathrm{~h}$ with $4 \%$ paraformaldehyde and washed for $3 \times 10 \mathrm{~min}$ with phosphate-buffered saline buffer. The kidney blocks were dehydrated and embedded in paraffin. The paraffin-embedded tissues were cut in $2-\mu \mathrm{m}$ sections on a rotary microtome (Leica Microsystems, Herlev, Denmark). The sections were deparaffinized and rehydrated. For immunoperoxidase labeling, endogenous peroxidase were blocked by $0.5 \% \mathrm{H}_{2} \mathrm{O}_{2}$ in absolute methanol for $10 \mathrm{~min}$ at room temperature. To reveal antigens, sections were incubated in $1 \mathrm{mmol} / \mathrm{l}$ Tris solution ( $\mathrm{pH} 9.0$ ) supplemented with $0.5 \mathrm{mmol} / \mathrm{l}$ ethyleneglycol-bis(baminoethylether)- N,N9-tetraacetic acid (EGTA) and heated using a microwave oven for $10 \mathrm{~min}$. Nonspecific binding of immunoglobulin $\mathrm{G}$ was prevented by incubating the sections in $50 \mathrm{mmol} / \mathrm{l} \mathrm{NH}_{4} \mathrm{Cl}$ for $30 \mathrm{~min}$, followed by blocking in PBS supplemented with $1 \%$ BSA, $0.05 \%$ saponin, and $0.2 \%$ gelatin. Sections were incubated overnight at $4{ }^{\circ} \mathrm{C}$ with primary antibodies diluted in PBS supplemented with $0.1 \%$ bovine serum albumin and $0.3 \%$ Triton X-100. After being rinsed with PBS supplemented with $0.1 \%$ bovine serum albumin, $0.05 \%$ saponin, and $0.2 \%$ gelatin for $3 \times 10 \mathrm{~min}$, the sections were incubated with horseradish peroxidase-conjugated secondary antibodies (P448, goat anti-rabbit immunoglobulin, DAKO, Denmark) diluted 1:200 in PBS supplemented with $0.1 \%$ bovine serum albumin and $0.3 \%$ Triton X-100 for $1 \mathrm{~h}$ at room temperature. After rinsing with PBS washing buffer, the sites of antibody-antigen reactions were visualized with $0.05 \%$ 
3, 3'-diaminobenzidine tetrachloride (Kem-en Tek, Copenhagen, Denmark) dissolved in distilled water with $0.1 \% \mathrm{H}_{2} \mathrm{O}_{2}$. The light microscope was carried out with Nikon Eclipse Ci microscope (Nikon, Tokyo, Japan).

\section{Primary Antibodies}

For immunohistochemistry, we used previously characterized polyclonal antibodies summarized as follows:

1. AQP1 (2353 AP fr 2-5): an affinity-purified antibody to AQP1 (37)

2. AQP2 (H7661 AP fr 2-5): an affinity-purified antibody to AQP2 (38).

\section{Hematoxylin and Eosin Staining}

Kidneys from G60, G80, and G100 fetal pigs were fixed, embedded in paraffin and cut into $2-\mu \mathrm{m}$ slides as described above. The slides were dewaxed in xylene, stained with haematoxylin and eosin and evaluated under a light microscope of Nikon Eclipse Ci microscope (Nikon).

\section{Statistical Analysis}

Statistical comparisons between sexes from G60, G80, and G100 group were performed using two-way ANOVA. A Tukey-Kramer multiple comparisons post hoc test was performed (Stata 12). $P<0.05$ was considered to indicate a significant difference. Values are presented as the mean \pm SEM.

\section{ACKNOWLEDGMENTS}

The expert technical assistance from Gitte Kall and Gitte Skou is highly appreciated. The authors thank Jens Christian Djurhuus and Jørgen Frøkiær for the support of the study.

\section{STATEMENT OF FINANCIAL SUPPORT}

This study was supported by the Karen Elise Jensen Foundation and the AP Moller Foundation.

Disclosure: None of the authors have conflicts of interest or financial relationships relevant to this article to disclose.

\section{REFERENCES}

1. Yamamoto T, Sasaki S, Fushimi K, et al. Expression of AQP family in rat kidneys during development and maturation. Am J Physiol 1997;272(2 Pt 2): F198-204.

2. Nielsen S, DiGiovanni SR, Christensen EI, Knepper MA, Harris HW. Cellular and subcellular immunolocalization of vasopressin-regulated water channel in rat kidney. Proc Natl Acad Sci USA 1993;90:11663-7.

3. Ervin MG, Kullama LK, Ross MG, Leake RD, Fisher DA. Vasopressin receptors and effects during fetal development. Regul Pept 1993;45:203-8.

4. Ross MG, Ervin MG, Leake RD, Fisher DA. Amniotic fluid ionic concentration in response to chronic fetal vasopressin infusion. Am J Physiol 1985;249(3 Pt 1):E287-91.

5. Ostrowski NL, Young WS III, Knepper MA, Lolait SJ. Expression of vasopressin V1a and V2 receptor messenger ribonucleic acid in the liver and kidney of embryonic, developing, and adult rats. Endocrinol 1993;133:1849-59.

6. Liu H, Wintour EM. Aquaporins in development - a review. Reprod Biol Endocrinol 2005;3:18.

7. Smith BL, Baumgarten R, Nielsen S, Raben D, Zeidel ML, Agre P. Concurrent expression of erythroid and renal aquaporin CHIP and appearance of water channel activity in perinatal rats. J Clin Invest 1993;92:2035-41.

8. Baum MA, Ruddy MK, Hosselet CA, Harris HW. The perinatal expression of aquaporin-2 and aquaporin-3 in developing kidney. Pediatr Res 1998;43:783-90.

9. Seifman BD, Rubin MA, Williams AL, Wolf JS Jr. Functional effects of unilateral laser papillectomy in the pig. Urology 2001;57:832-6.

10. Nygard AB, Jørgensen CB, Cirera S, Fredholm M. Selection of reference genes for gene expression studies in pig tissues using SYBR green $\mathrm{qPCR}$. BMC Mol Biol 2007;8:67.
11. Parreira KS, Debaix H, Cnops Y, Geffers L, Devuyst O. Expression patterns of the aquaporin gene family during renal development: influence of genetic variability. Pflugers Arch 2009;458:745-59.

12. Devuyst O, Burrow CR, Smith BL, Agre P, Knepper MA, Wilson PD. Expression of aquaporins-1 and -2 during nephrogenesis and in autosomal dominant polycystic kidney disease. Am J Physiol 1996;271(1 Pt 2):F169-83.

13. Wintour EM, Earnest L, Alcorn D, Butkus A, Shandley L, Jeyaseelan K. Ovine AQP1: cDNA cloning, ontogeny, and control of renal gene expression. Pediatr Nephrol 1998;12:545-53.

14. Butkus A, Earnest L, Jeyaseelan K, et al. Ovine aquaporin-2: cDNA cloning, ontogeny and control of renal gene expression. Pediatr Nephrol 1999;13:379-90.

15. Moritz KM, Wintour EM. Functional development of the meso- and metanephros. Pediatr Nephrol 1999;13:171-8.

16. Herak-Kramberger CM, Breljak D, Ljubojević M, et al. Sex-dependent expression of water channel AQP1 along the rat nephron. Am J Physiol Renal Physiol 2015;308:F809-21.

17. Mahler B, Kamperis K, Ankarberg-Lindgren C, Frøkiær J, Djurhuus JC, Rittig S. Puberty alters renal water handling. Am J Physiol Renal Physiol 2013;305:F1728-35.

18. Ervin MG, Ross MG, Leake RD, Fisher DA. V1- and V2-receptor contributions to ovine fetal renal and cardiovascular responses to vasopressin. Am J Physiol 1992;262(4 Pt 2):R636-43.

19. Nielsen S, Chou CL, Marples D, Christensen EI, Kishore BK, Knepper MA. Vasopressin increases water permeability of kidney collecting duct by inducing translocation of aquaporin-CD water channels to plasma membrane. Proc Natl Acad Sci USA 1995;92:1013-7.

20. Yamamoto T, Sasaki S, Fushimi K, et al. Vasopressin increases AQP-CD water channel in apical membrane of collecting duct cells in Brattleboro rats. Am J Physiol 1995;268(6 Pt 1):C1546-51.

21. Kortenoeven ML, Fenton RA. Renal aquaporins and water balance disorders. Biochim Biophys Acta 2014;1840:1533-49.

22. Liu J, Sharma N, Zheng W, et al. Sex differences in vasopressin $V_{2}$ receptor expression and vasopressin-induced antidiuresis. Am J Physiol Renal Physiol 2011;300:F433-40.

23. Wang YX, Edwards RM, Nambi P, et al. Sex difference in the antidiuretic activity of vasopressin in the rat. Am J Physiol 1993;265(6 Pt 2):R1284-90.

24. Dickinson H, Moritz KM, Kett MM. A comparative study of renal function in male and female spiny mice - sex specific responses to a high salt challenge. Biol Sex Differ 2013;4:21.

25. Cheema MU, Irsik DL, Wang Y, et al. Estradiol regulates AQP2 expression in the collecting duct: a novel inhibitory role for estrogen receptor $\alpha$. Am J Physiol Renal Physiol 2015;309:F305-17.

26. Reyes FI, Boroditsky RS, Winter JS, Faiman C. Studies on human sexual development. II. Fetal and maternal serum gonadotropin and sex steroid concentrations. J Clin Endocrinol Metab 1974;38:612-7.

27. Wang YX, Crofton JT, Share L. Sex differences in the cardiovascular and renal actions of vasopressin in conscious rats. Am J Physiol 1997;272(1 Pt 2):R370-6.

28. Rittig S, Schaumburg HL, Siggaard C, Schmidt F, Djurhuus JC. The circadian defect in plasma vasopressin and urine output is related to desmopressin response and enuresis status in children with nocturnal enuresis. J Urol 2008;179:2389-95.

29. Stachenfeld NS, Splenser AE, Calzone WL, Taylor MP, Keefe DL. Sex differences in osmotic regulation of AVP and renal sodium handling. J Appl Physiol (1985) 2001;91:1893-901.

30. Murillo-Carretero MI, Ilundáin AA, Echevarria M. Regulation of aquaporin mRNA expression in rat kidney by water intake. J Am Soc Nephrol 1999;10:696-703.

31. Tamma G, Goswami N, Reichmuth J, De Santo NG, Valenti G. Aquaporins, vasopressin, and aging: current perspectives. Endocrinol 2015;156:777-88.

32. Fenton RA, Pedersen CN, Moeller HB. New insights into regulated aquaporin-2 function. Curr Opin Nephrol Hypertens 2013;22:551-8.

33. Silbiger SR, Neugarten J. The impact of gender on the progression of chronic renal disease. Am J Kidney Dis 1995;25:515-33.

34. Kang SW, Kim YW, Kim YH, Sohn HS, Joo H, Kim E. Study of the association of -667 aquaporin-2 (AQP-2) A/G promoter polymorphism with the 
incidence and clinical course of chronic kidney disease in Korea. Ren Fail 2007;29:693-8.

35. Xing L, Nørregaard R. The impact of gender on AQP1-3 expression in response to neonatal partial unilateral ureteral obstruction. Int J Nephrol Kidney Failure 2015;1:1-8.

36. Pfaffl MW. A new mathematical model for relative quantification in realtime RT-PCR. Nucleic Acids Res 2001;29:e45.
37. O’Neill H, Lebeck J, Collins PB, Kwon TH, Frøkiaer J, Nielsen S. Aldosterone-mediated apical targeting of $\mathrm{ENaC}$ subunits is blunted in rats with streptozotocin-induced diabetes mellitus. Nephrol Dial Transplant 2008;23:1546-55.

38. Nielsen J, Kwon TH, Praetorius J, Frøkiaer J, Knepper MA, Nielsen S. Aldosterone increases urine production and decreases apical AQP2 expression in rats with diabetes insipidus. Am J Physiol Renal Physiol 2006;290:F438-49. 